\title{
Oligomer Composition and Oxygen Binding of the Hemocyanin of the Blue Crab Callinectes sapidus
}

\author{
CP Mangum \\ J Greaves \\ Virginia Institute of Marine Science \\ JS Rainer \\ Virginia Institute of Marine Science
}

Follow this and additional works at: https://scholarworks.wm.edu/vimsarticles

Part of the Aquaculture and Fisheries Commons

\section{Recommended Citation}

Mangum, CP; Greaves, J; and Rainer, JS, "Oligomer Composition and Oxygen Binding of the Hemocyanin of the Blue Crab Callinectes sapidus" (1992). VIMS Articles. 1696.

https://scholarworks.wm.edu/vimsarticles/1696 


\title{
Oligomer Composition and Oxygen Binding of the Hemocyanin of the Blue Crab Callinectes sapidus
}

\author{
CHARLOTTE P. MANGUM ${ }^{1}$, JOHN GREAVES ${ }^{2}$, AND JULIA S. RAINER ${ }^{2}$ \\ 'Department of Biology, College of William and Mary, Williamsburg, Virginia 23185 \\ and ${ }^{2}$ School of Marine Science, Virginia Institute of Marine Science, \\ College of William and Mary, Gloucester Point, Virginia 23062
}

\begin{abstract}
In the blue crab, the ratio of hexamers to dodecamers of the $\mathrm{O}_{2}$ carrier hemocyanin varies in natural populations. Isolated dodecamers have a lower $\mathrm{O}_{2}$ affinity and greater cooperativity than isolated hexamers. The difference in $\mathrm{O}_{2}$ binding can also be resolved in native mixtures that differ in polymer composition. A high content of dodecamers in native mixtures is, in fact, correlated with the presence of an invariant polypeptide chain that is believed to link two hexamers to form dodecamers. On the other hand, the content of a variable chain that has been postulated to play a role in hexamer pairing is correlated with a low content of dodecamers. The variable, but not the invariant, monomers can be present in levels so low that they must not be represented in all dodecamers in the blood.
\end{abstract}

\section{Introduction}

The hemocyanin $(\mathrm{Hc})$ of the blue crab Callinectes sapidus Rathbun, like that of most crustaceans, exists in the blood as a mixture of hexamers $(1 \times 6)$ and dodecamers $(2 \times 6)$. The two oligomers are stable aggregates, not members of a chemical equilibrium. Oligomerization is important in optimizing the physical properties of the blood (Snyder and Mangum, 1982; Mangum, 1986) but its respiratory significance, if any, is not known. While both Brouwer et al. (1982) and Johnson et al. (1984) mentioned that hexamers and dodecamers do not differ in $\mathrm{O}_{2}$ binding, neither reported the data. Brouwer (pers. comm.) has kindly communicated his opinion that the finding was not definitive because the two oligomers were

Received 20 June 1991; accepted 8 August 1991. poorly separated in their experiments by atmospheric pressure permeation gel chromatography.

In $C$. sapidus, the Hc oligomers are built of five to six different polypeptide chains, four of which (in our terminology, Nos. 1, 3, 5, and 6) exhibit considerable quantitative variability in natural populations. In the laboratory, changes in the concentrations of two to three of the four (Nos. 3, 5, and 6) can be induced by prolonged hypoxia or a change in acclimation salinity. Accompanying the alterations in monomeric subunit composition are highly adaptive changes in intrinsic $\mathrm{O}_{2}$ affinity, which can be specifically attributed to the levels of chains 3 and 6 (Mason et al., 1983; Mangum and Rainer, 1988; Rainer, 1989; deFur et al., 1990).

The nature of the hexamers that do not form dodecamers, but remain as such in the blood, is unclear. Hamlin and Fish (1977) described them as comprising "as much as" $20 \%$ of the total material in their purified Hc preparations. Brouwer et al. (1982) and Johnson et al. (1984) mentioned similar figures. Herskovits et al. (1981), however, specified a range of $30-50 \%$ and raised the possibility of a seasonal change. Recently, we have found that the ratio does in fact vary in nature (Greaves et al., 1991).

On the basis of a combination of electrophoretic and immunological properties, Markl (1986, for review) has classified the monomeric chains of the arthropod Hes into four categories that differ in their interspecific variability and their putative role in the assembly of the native polymers. The chain (No. 4) that is believed to serve as the dodecamer-former in C. sapidus (Stöcker et al., 1988) is one of the two essentially invariant ones in our ongoing sample, which presently totals about 1000 individuals (Rainer, 1989; Mangum, 1990). Densitometric scans shown by Johnson et al. (1984), however, indicate that 
the concentration of chain 4 was at least as high in a hexameric fraction as in a dodecameric fraction. Moreover, their hexameric fraction lacked all but perhaps trace quantities of chains 5 and 6 , two of the most variable ones in nature. They suggested that these chains, presumably instead of chain 4 , play a role in dodecamer formation.

The way in which the variable chains influence $\mathrm{O}_{2}$ binding is presently unclear. Those that do not play an important role in hexamer pairing (c.g., No. 3) may have simple and direct effects on $\mathrm{O}_{2}$ affinity. Chains that do participate in hexamer pairing, however, could exert their influence indirectly if $\mathrm{O}_{2}$ binding of the two oligomers differs.

We attempted to circumvent the problem of poor separation at atmospheric pressure by developing a protocol for HPLC, which proved to achieve clean separation (Greaves et al., 1992). In the present investigation we used the procedure 1) to examine the respiratory properties of the two fractions and 2) to correlate the ratio of $2 \times 6: 1$ $\times 6$ aggregates in native mixtures with their subunit composition and $\mathrm{O}_{2}$ binding. We also tried, without success, to compare the respiratory properties of native hexamers with those of hexamers prepared as dissociation products of dodecamers.

\section{Materials and Methods}

Large adult males ( $\geq 80 \mathrm{~g}$ wet wt) were obtained from either the seaside (Eastern Shore of Virginia at Wachapreague) or estuarine (York R.) populations studied earlier (Mason et al., 1983; Mangum and Rainer, 1988; Rainer, 1989). Blood samples were taken from the infrabranchial sinuses and declotted as previously described. In the present investigation the samples were also filtered at $0.22 \mu \mathrm{m}$ for injection into the HPLC system.

\section{HPLC}

HPLC was carried out on a Perkin Elmer Series 4 system equipped with one or two (connected in series) Ultrahydrogel 1000 size exclusion columns $(7.8 \times 300 \mathrm{~mm}$; Waters Associates). The mobile phase was a saline containing $300 \mathrm{mmol}^{-1} \mathrm{NaCl}, 25 \mathrm{mmol} 1^{-1} \mathrm{MgCl}_{2}$, and 10 $\mathrm{mmol} 1^{-1} \mathrm{CaCl}_{2}$; this saline was also filtered at $0.22 \mu \mathrm{m}$ immediately prior to use. The Perkin Elmer Model LC95 variable wavelength $U V$ absorbance detector was set at 337 and $280 \mathrm{~nm}$. Further details of the protocol were described by Greaves et al. (1992); an example of the separation is shown as Figure 1.

\section{$\mathrm{O}_{2}$ binding}

These measurements were performed on the fractions separated by HPLC, after reconcentration by membrane

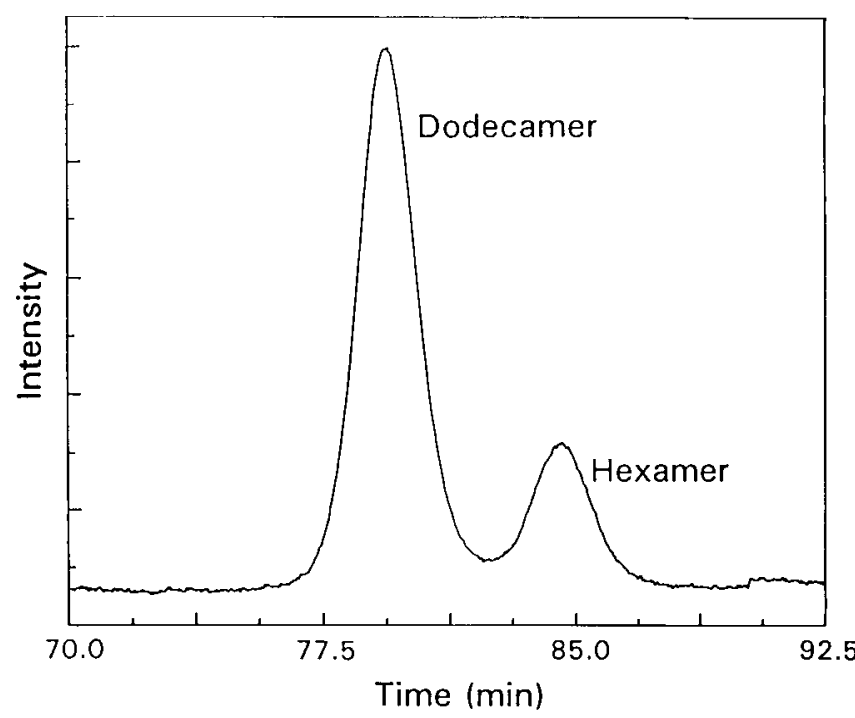

Figure 1. An example of the separation of oligomers obtained by HPLC. Absorbance was monitored at $337 \mathrm{~nm}$.

centrifugation (Centricon 30), or on unfractionated aliquots of the same samples analyzed by HPLC, following dialysis against $0.05 \mathrm{~mol} \mathrm{l}^{-1}$ Tris maleate buffered saline (specified above). Because reconcentration is a lengthy process, aliquots of the isolated oligomers were examined as soon as the $\mathrm{Hc}$ levels reached an absorbance at $337 \mathrm{~nm}$ of $0.25-0.58$ (2-3 h centrifugation), using a tonometric procedure suitable for dilute samples. Precision mixed, humidified gases were passed across rapidly shaken samples at constant temperature and atmospheric pressure, and the changes in absorbance at $337 \mathrm{~nm}$ determined (procedure described in detail by Mason et al., 1983) with a Mervyn Roy 501 spectrophotometer. Rather than dissociate the remainder of the available material for electrophoresis, we chose to use the cell respiration procedure (Mangum and Lykkeboe, 1979) to document more extensively the preliminary findings of the tonometry by performing repeated $\mathrm{O}_{2}$ binding measurements on the two intact oligomers. The procedure required further reconcentration for a total of 7-8 h. Native mixtures of the two oligomers, aliquots of which were analysed by electrophoresis, were also examined using this procedure. These samples were dialyzed overnight against buffered saline.

\section{Electrophoresis}

Samples were dialyzed overnight against $50 \mathrm{mmol} \mathrm{l}^{-1}$ Tris $\mathrm{HCl}$ (pH 8.9) containing $10 \mathrm{mmol}^{-1}$ EDTA to dissociate the native oligomers into their monomeric subunits. Alkaline dissociation electrophoresis was carried out, as described by Mangum and Rainer (1988). The gels (12\%) were scanned with a Shimadzu densitometer. 


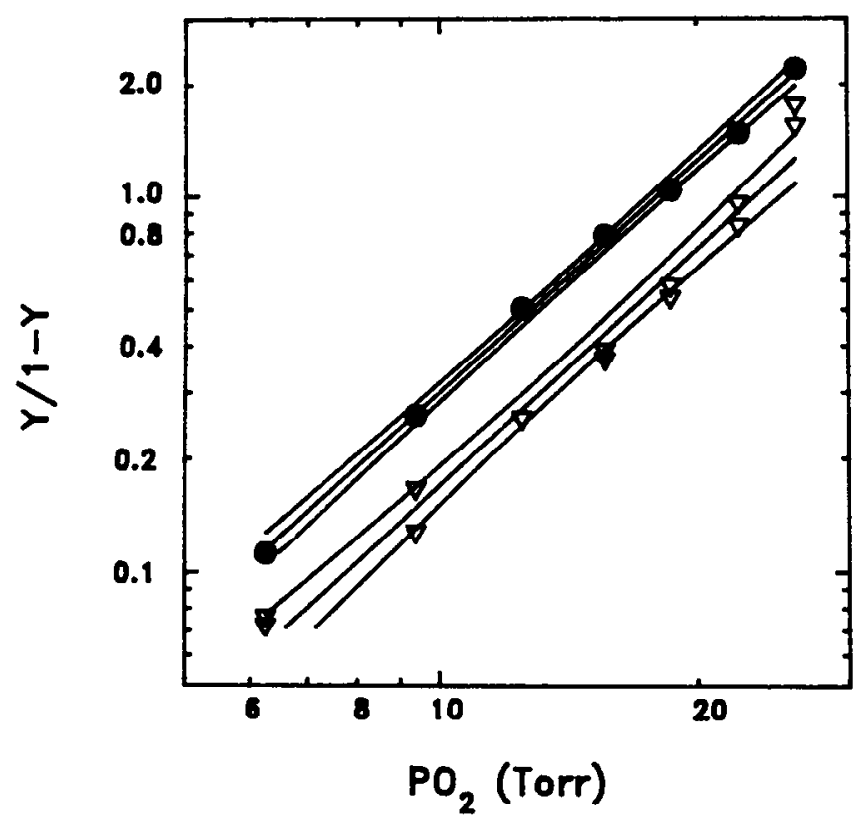

Figure 2. Hill plots of $\mathrm{O}_{2}$ binding properties of isolated hexamers and dodecamers. $25^{\circ} \mathrm{C}, 0.05 \mathrm{~mol}^{-1}$ Tris buffered saline containing 10

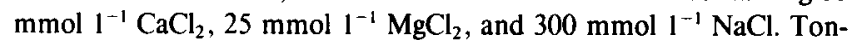
ometric data for one aliquot of hexamers (circles, $\mathrm{pH} 7.49$ ) and two of dodecamers (triangles, $\mathrm{pH} 7.52$ ). Lines are regression lines and their $95 \%$ confidence intervals.

\section{Results}

\section{HPLC}

We did not observe the material larger than the about $900 \mathrm{kD}$ dodecamers that had been reported by both Hamlin and Fish (1977) and Herskovits et al. (1981). Because this material (which has a sedimentation coefficient of 33S) should have a molecular weight in excess of the separation range of our gel, it should have appeared as a shoulder on the peak of the dodecameric fraction. Moreover, as pointed out earlier (Greaves et al., 1992), the ratios of the 280:337 nm areas, obtained for the two oligomer fractions and for the samples from different individuals, were statistically indistinguishable, despite the variation in contents of the two oligomers described below. The optical evidence, then, indicates that no material other than $\mathrm{Hc}$ was present and that the condition of the active site of the two oligomers does not differ.

\section{$\mathrm{O}_{2}$ binding}

The results obtained in three tonometric determinations suggest that isolated dodecamers have a significantly lower $\mathrm{O}_{2}$ affinity than isolated hexamers (Fig. 2), despite a slightly higher experimental $\mathrm{pH}$. The $95 \%$ confidence intervals do not overlap even though the fit of regression lines to the data for dodecamers is poor because, like many native mixtures of crustacean Hcs, they show an increase in cooperativity at higher oxygenation states. Because of the poor fit, cooperativity $\left(n_{50}\right)$ was estimated in Figure 3 from regression analysis of only the points $(n=6)$ in Figure 2 that exceed $30 \% \mathrm{HcO}_{2}$; the available data in this region suggest that dodecamers have a significantly $(P$ $<.015)$ higher cooperativity than hexamers. The cell respiration procedure, with which a larger number of determinations was made (Fig. 3), yielded very similar results: the hexameric fraction has a significantly higher $\mathrm{O}_{2}$ affinity ( $P=.0325$ according to Student's $t$ test) and lower cooperativity $(P=.0495)$. Neither mean value for $\mathrm{O}_{2}$ affinity of the isolated oligomers differs significantly from the intermediate value for the native, unfractionated mixture ( $P=.25$ for the dodecamers and .12 for the hexamers) although, following the dilution, fractionation and reconcentration, cooperativity had clearly fallen in both cases $(P<.0005)$. The value of $\mathrm{n}_{50}$ for the native mixture, which had not been diluted, fractionated, and reconcentrated, is typical of this species under the experimental conditions
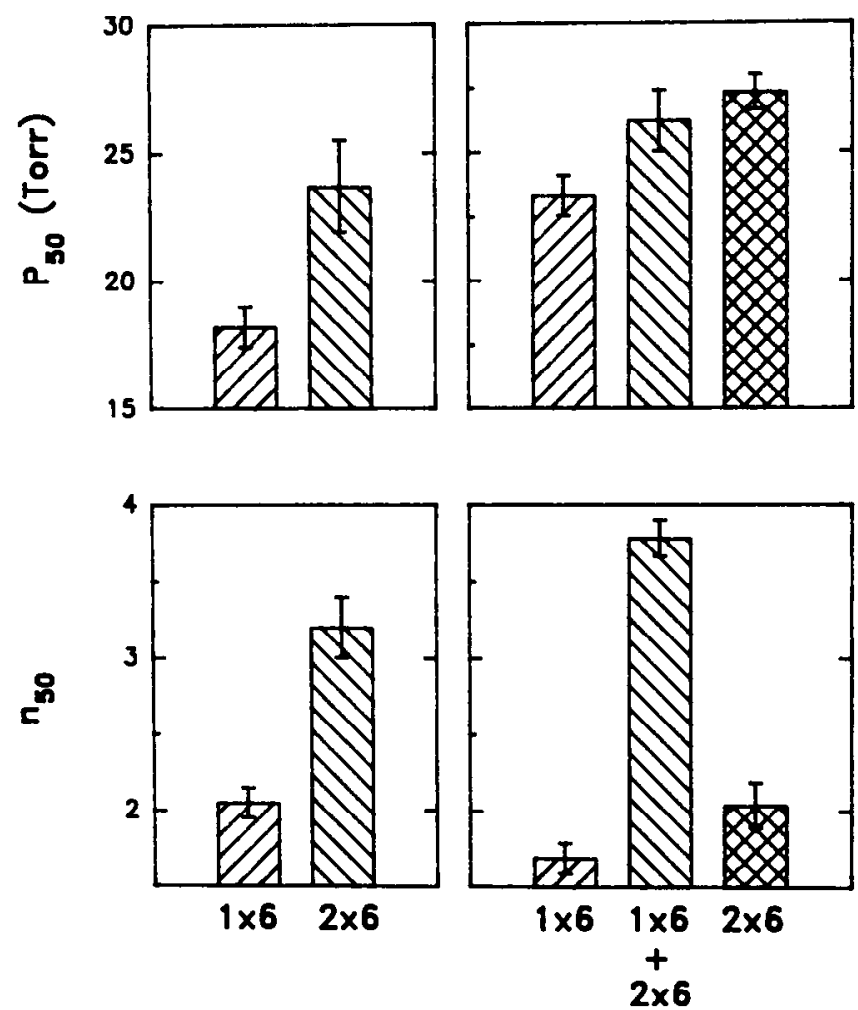

Figure 3. $\mathrm{O}_{2}$ binding of isolated hexamers and dodecamers. $25^{\circ} \mathrm{C}$. Panels at left show tonometric data ( $\mathrm{pH} 7.49-7.52)$ from Figure 1, with error as $95 \%$ confidence intervals. Panels at right show cell respiration data for 6 replicate measurements of isolated hexamers ( $\mathrm{pH} 7.40$ ), dodecamers ( $\mathrm{pH} 7.40$ ), and the dialyzed but unfractionated $(77 \%$ dodecamers) sample ( $\mathrm{pH} 7.38$ ) from which the two fractions were prepared. Buffered saline as in Figure 1. Mean values \pm S.E. 
employed here (e.g., Mason et al., 1983; Mangum and Rainer, 1988).

The $\mathrm{O}_{2}$ binding properties of two undiluted and unfractionated samples with a high content of dodecamers (Fig. 4) are indistinguishable from one another (according to Student's $t$ test, $P=.2$ for both $\mathrm{P}_{50}$ and $\mathrm{n}_{50} ; \mathrm{n}=6$ ); similarly, two with a low content of dodecamers had indistinguishable $\mathrm{O}_{2}$ binding properties $(P>.4 ; \mathrm{n}=6)$. The $\mathrm{O}_{2}$ affinity of each pair differs significantly from that of the alternative pair $(P=.004)$; specifically, a high content of dodecamers is accompanied by a lower affinity. The cooperativity values, which are well within the range usually observed in this species, are also significantly greater in the sample with the higher dodecamer content $(P=.005)$.

We attempted to create artificial hexamers by freezing and thawing (11X over a two-day period) unreconcentrated dodecamers. Following membrane centrifugation, all $\mathrm{O}_{2}$ binding activity had disappeared in this fraction, as well as that containing native hexamers. We then examined the polymer composition and $\mathrm{O}_{2}$ binding of the remainder of the unfractionated and never diluted sample from which the two fractions had been prepared, which had also been frozen and thawed 11 times. Prior to freezing, $77 \%$ of this sample consisted of dodecamers, and it had the $\mathrm{O}_{2}$ binding properties shown in Figure 2. Following freezing and thawing, $76 \%$ consisted of dodecamers, and it exhibited an indistinguishable $\mathrm{O}_{2}$ affinity and cooperativity $(P=.63)$.

\section{Subunit composition}

The monomer and oligomer compositions of samples from 13 individuals collected in the summer from the seaside population are shown in Table I. After first transforming the percentages to a normal distribution (arcsin), we examined the relationship between the quantities of each chain and the content of dodecamers by linear regression analysis. There is no indication of a correlation

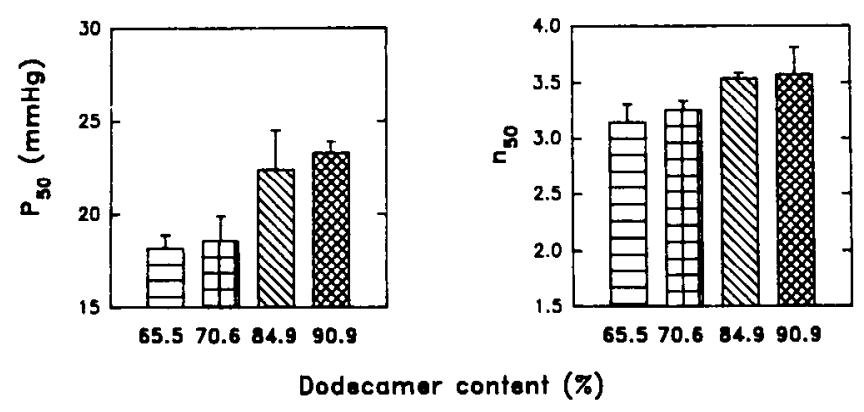

Figure 4. $\mathrm{O}_{2}$ binding properties (cell respiration procedure) of native Hcs with different contents of dodecamers. $25^{\circ} \mathrm{C}$, pH 7.50. Buffered saline as in Figure 1. Mean \pm S.E. $(n=6)$.
Table I

Subunit and oligomer composition of hemocyanins from 13 individuals of Callinectes sapidus

\begin{tabular}{|c|c|c|c|c|c|c|c|}
\hline \multirow[b]{2}{*}{ No. } & \multicolumn{6}{|c|}{$\%$ Total peak area* } & \multirow{2}{*}{$\begin{array}{c}\% \\
2 \times 6 \text {-mers }\end{array}$} \\
\hline & 1 & 2 & 3 & 4 & 5 & 6 & \\
\hline 1 & 8.4 & 27.2 & 5.4 & 20.0 & 26.2 & 12.7 & 65.5 \\
\hline 2 & 23.4 & 28.2 & 7.0 & 24.8 & 9.3 & 7.0 & 70.6 \\
\hline 3 & 10.8 & 22.2 & 9.8 & 25.4 & 14.9 & 16.9 & 74.4 \\
\hline 4 & 9.7 & 29.0 & 6.8 & 32.7 & 12.2 & 9.6 & 78.3 \\
\hline 5 & 17.3 & 19.6 & 19.5 & 23.4 & 5.3 & 14.9 & 79.6 \\
\hline 6 & 12.5 & 31.8 & 11.0 & 23.9 & 8.3 & 12.3 & 81.1 \\
\hline 7 & 9.3 & 24.7 & 15.2 & 26.8 & 12.0 & 11.9 & 82.5 \\
\hline 8 & 5.8 & 20.0 & 10.8 & 34.6 & 12.1 & 16.7 & 84.4 \\
\hline 9 & 9.2 & 32.3 & 7.1 & 34.1 & 4.0 & 13.3 & 84.4 \\
\hline 10 & 23.0 & 23.8 & 10.0 & 28.7 & 9.0 & 5.8 & 84.9 \\
\hline 11 & 17.4 & 21.0 & 9.7 & 29.4 & 10.2 & 12.3 & 85.1 \\
\hline 12 & 5.1 & 29.8 & 9.1 & 31.1 & 10.4 & 14.3 & 86.8 \\
\hline 13 & 5.4 & 24.5 & 13.5 & 25.5 & 6.1 & 15.2 & 88.0 \\
\hline
\end{tabular}

* Figures less than $1 / 12$ th of the dodecameric fraction shown in italics.

between the variation in levels of chains $1,2,3$, or 6 and the content of dodecamers $(\mathrm{r}=-.103$ to $.421 ; P>.10)$. However, dodecamer content is highly and directly correlated with relative content of chain $4(\mathrm{r}=.601 ; P=.02)$ (Fig. 5). Both of the two invariant chains ( 2 and 4 ), unlike variable ones, are present in sufficient quantities to be represented in each hexamer of a $2 \times 6$ pair. The correlation with dodecamer content is not improved, however, by summing the relative contents of the chains 2 and 4 ( $\mathrm{r}=.341 ; P>.10)$. Dodecamer content is highly but inversely correlated with the levels of chain $5(\mathrm{r}=-.653$; $P=.01)$.

The numerical relationship between the $\%$ composition values for the two parameters is also of interest. Any chain present in less than the figure [(its \% composition) $/(\%$ dodecamers) $=1 / 12$ th or $] 8.3 \%$ must not be represented in all dodecamers. All four variable (but neither of the invariant) chains can fall into this category, some trivially and probably not outside of the error of densitometry, but some by a large margin (Table I).

\section{Discussion}

Morris (1988) systematically investigated the effects of freezing on crustacean Hcs. Repeated bouts of freezing and thawing resulted in progressive dissociation of dodecamers to hexamers, although to a different degree in the four species examined, and an attendant decrease in cooperativity. As noted by Morris (1988), much of our own experience concurs with his: thawed crustacean Hcs often show impaired cooperativity (see also Reese, 1989). Because freezing (at least using high concentrations and freezing for only a brief period) had no dis- 

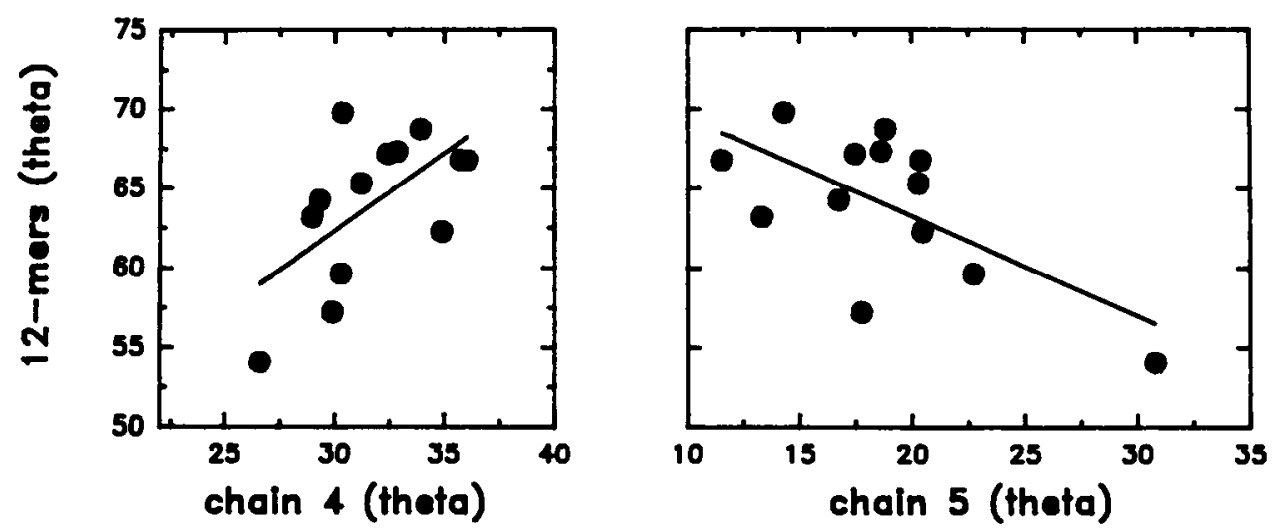

Figure 5. The relationship between the fractional composition of dodecamers and chains 4 and 5 of Callinecles sapidus $\mathrm{Hc}$, expressed as arcsin transformed percentages. Lines are fitted regression lines.

cernible effect on C. sapidus $\mathrm{Hc}$, we can only conclude that it is more stable than those examined by Morris (1988).

The present data provide further information on the natural variability of the two native oligomers in this species. They provide no evidence that chain 6 plays a role in the pairing of hexamers. However, our data strongly support the inference of Stöcker et al. (1988) that high levels of chain 4 promote dodecamer formation. Moreover, they do not support the conclusion reached by Johnson et al. (1984) that chain 5 promotes dodecamer formation.

We should note that, in the present sample, chain 1 appears to be more variable than 3 or 6 and almost as variable as 5 . Although we have noticed the variability of this chain in much larger samples (Rainer, 1989), we have not previously found nearly as much variability, and we suggest that the present sample is misleading because of its small size. In addition, we have no evidence that chain 1 responds to an environmental stimulus or that it influences respiratory properties. The high and constant levels of chain 2 are intriguing because this monomer is not known to play a role in oligomerization. According to Markl's classification, it is an interspecifically conservative type of chain, but chain 3 (Stocker et al., 1988), which is highly variable intraspecifically, is also classified as conservative interspecifically.

Our findings demonstrate different $\mathrm{O}_{2}$ binding properties in the two native oligomers: dodecamers, the predominant aggregate in the blood, have a lower $\mathrm{O}_{2}$ affinity and greater cooperativity than hexamers. The difference is detectable in both isolated fractions and native mixtures of different proportions of the two. The variable chain No. 5, which is not known to influence $\mathrm{O}_{2}$ affinity directly, could have an indirect influence via inhibition of hexamer pairing. On the other hand, the variable chains 3 and 6, which influcnec $\mathrm{O}_{2}$ affinity, are not corrclated with dodecamer content. The most likely inference at present is that their respiratory effect is simple and direct.

Finally, Johnson et al. (1984) concluded that the levels of chains 5 and 6 indicate that they comprise $1 / 6$ th of the dodecamers. The present data indicate that they (as well as chain 1) may occur in either considerably higher or lower levels, which suggests heterogeneity of the dodecamers in the blood. In fact, some heterogeneity would be expected if the changes in subunit composition that are responsible for the observed adaptability of respiratory properties of the $\mathrm{Hc}$ are physiologically labile.

\section{Acknowledgments}

Supported by NSF DCB 88-16172 (Physiological Processes) to CPM. We thank D. A. Scholnick for performing the tonometric measurements.

\section{Literature Cited}

Brouwer, M., C. Bonaventura, and J. Bonaventura. 1982. Heavy metal ion interactions with Callinectes sapidus hemocyanin: structural and functional changes induced by a variety of heavy metal ions. Blochemistry 21: 2529-2538.

de Fur, P. L., C. P. Mangum, and J. E. Reese. 1990 . Respiratory responses of the blue crab Callinectes sapidus to long-term hypoxia. Biol. Bull. 178: 46-54.

Greaves, J., J. S. Rainer, and C. P. Mangum. 1992. Size-exclusion high performance liquid chromatography of the dodecameric and hexameric forms of hemocyanin from Callinectes sapidus. Mar. Biol. (in press).

Herskovits, T. T., L. J. Erhunmwunsee, R. C. San George, and A. Herp. 1981. Subunit structure and dissociation of Callinectes sapidus hemocyanin. Biochim. Biophys. Acta 667: 44-58.

Hamlin, L. M., and W. W. Fish. 1977. The subunit characterization of Callinectes sapidus hemocyanin. Biochim. Biophys. Acta 491: 46-52.

Johnson, B. A., C. Bonaventura, and J. Bonaventura. 1984. Allosteric modulation of Callinectes sapidus hemocyanin by binding of Llactate. Biochemistry 23: 872-878. 
Mangum, C. P. 1986. Osmoregulation in marine and estuarine animals: the view of a respiratory physiologist. Boll. Zool. 53: 1-8.

Mangum, C. P. 1990. Inducible $\mathrm{O}_{2}$ carriers in the crustaceans. Pp. 92 103 in Animal Nutrition and Transport Processes, J-P. Truchot and B. Lahlou, eds. Karger, Basel.

Mangum, C. P., and G. Lykkeboe. 1979. The influence of inorganic ions and $\mathrm{pH}$ on the oxygenation properties of the blood in the gastropod mollusc Busycon canaliculatum. J. Exp. Zool. 207: 417-430.

Mangum, C. P., and J. Rainer. 1988. The relationship between subunit composition and $\mathrm{O}_{2}$ binding of blue crab hemocyanin. Bial $R_{4}$ ll. 174: $77-82$.

Markl, J. 1986. Evolution of functionally and structurally diverse subunits in the respiratory protein hemocyanin from arthropods. Biol. Aull. 171: 90-115.

Mason, R. P., C. P. Mangum, and G. Godette. 1983. The influence of inorganic ions and acclimation salinity on hemocyanin-oxygen binding in the blue crab Callinectes sapidus. Butl. 164: 104-123.
Morris, S. 1988. Effects of freezing on the function and association state of crustacean haemocyanins. J. Exp. Biol. 138: 535-539.

Rainer, J. S. 1989. Distribution of the heterogeneous hemocyanin subunits along a salinity gradient. M.A. Thesis, College of William and Mary, Williamsburg, Virginia. $62 \mathrm{pp}$.

Reese, J. E. 1989. Structure and function of crustacean hemocyanins. M.A. Thesis, College of William and Mary, Williamsburg, Virginia. $75 \mathrm{pp}$.

Snyder, G. K., and C. P. Mangum. 1982. The relationship between the size and shape of an extracellular oxygen carrier and the capacity for oxygen transport. Pp. 173-188 in Physiology and Biology of Horseshoe Crabs, J. Bonaventura, C. Bonaventura, and S. Tesh, eds. Alan R. Liss Inc., New York.

Stöcker, W., U. Raeder, M. M. C. Bijlholt, T. Wichertjes, E. F. J. van Bruggen, and J. Markl. 1988. The quaternary structure of four crustacean two-hexameric hemocyanins: immunocorrelation, stoichiometry, reassembly and topology of individual subunits. J. Comp. Physiol. B158: 271-289. 